Supplementary Information for

\title{
Mode Specific $S_{\mathrm{N}} 2$ Reaction Dynamics
}

\author{
Yan Wang, ${ }^{1,2,+}$ Hongwei Song, ${ }^{1,+}$ István Szabó, ${ }^{3}$ \\ Gábor Czakó, 3,* Hua Guo ${ }^{4, *}$ and Minghui Yang ${ }^{1, *}$
}

1 Key Laboratory of Magnetic Resonance in Biological Systems, National Center for Magnetic Resonance in Wuhan, State Key Laboratory of Magnetic Resonance and Atomic and Molecular Physics, Wuhan Institute of Physics and Mathematics, Chinese Academy of Sciences, Wuhan 430071, China

2 School of Chemical and Environmental Engineering, Hubei University for Nationalities, Enshi 445000, China

3 Department of Physical Chemistry and Materials Science, Institute of Chemistry, University of Szeged, Rerrich Béla tér 1, Szeged, H-6720, Hungary

4 Department of Chemistry and Chemical Biology, University of New Mexico, Albuquerque, New Mexico, 87131, USA

${ }^{+}$These authors contributed equally to this work.

*Authors to whom correspondence should be addressed. Electronic addresses: gczako@chem.uszeged.hu (GC), hguo@unm.edu (HG) and yangmh@wipm.ac.cn (MY). 


\section{S-I. Theory}

\section{S-I A. Quantum Dynamics}

In this work, a six-dimensional (6D) quantum dynamics (QD) model is developed for the title $\mathrm{S}_{\mathrm{N}} 2$ reaction. Modified from the original model of Palma and Clary, ${ }^{1}$ this reduceddimensional model for the $\mathrm{X}+\mathrm{YCZ}_{3} \rightarrow \mathrm{XY}+\mathrm{CZ}_{3}$ type reactions constrains the $\mathrm{YCZ}_{3}$ moiety to $C_{3 \mathrm{v}}$ symmetry. The Palma-Clary model has been successfully used to study quantum dynamics of several such reactions. ${ }^{2-9}$ As shown in Figure $\mathrm{S} 1$, the six coordinates for the $\mathrm{F}^{-}+\mathrm{CH}_{3} \mathrm{Cl}$ reaction include $\mathbf{R}$ as the vector from the center of mass of $\mathrm{CH}_{3} \mathrm{Cl}$ to $\mathrm{F}^{-}$, which also serves as the z-axis of the body-fixed (BF) frame, $\mathbf{r}$ as the vector from the center of mass of $\mathrm{CH}_{3}$ to $\mathrm{Cl}$, which coincide with the $C_{3 \mathrm{v}}$ symmetric axis $\boldsymbol{S}$, the symmetric $\mathrm{CH}$ stretching mode and the umbrella mode of the $\mathrm{CH}_{3}$ group described by scaled-polar coordinates $(q, \gamma)$, and the rotation of $\mathrm{CH}_{3} \mathrm{Cl}$ in the BF frame described by angles $(\theta, \varphi)$. The scaled-polar coordinates for the $\mathrm{CH}_{3}$ group are defined as $q=\sqrt{x^{2}+y^{2}}$ and $\gamma=\pi-\arctan (x / y)$ with $y=\sqrt{m_{C} /\left(m_{C}+3 m_{H}\right)} s$, where $x$ is the distance between atom $\mathrm{H}$ and the $C_{3 \mathrm{v}}$ symmetry axis $\boldsymbol{S}$ and $s$ is the distance between atom $\mathrm{C}$ and the center of three $\mathrm{H}$ atoms. As previous QCT studies of the $\mathrm{F}^{-}+\mathrm{CH}_{3} \mathrm{Cl}$ reaction have showed, ${ }^{10}$, ${ }^{11}$ a typical reactive scattering involves $\mathrm{F}^{-}$attacking $\mathrm{CH}_{3} \mathrm{Cl}$ from the $\mathrm{H}_{3} \mathrm{C}$ side, leading to the transition state with the $\mathrm{Cl}$ atom in the $C_{3}$ symmetry axis of $\mathrm{CH}_{3}$. Thus, the present $6 \mathrm{D}$ model is expected to give a reasonable description of the title reaction.

The 6D model Hamiltonian for the $\mathrm{F}^{-}+\mathrm{CH}_{3} \mathrm{Cl}$ reaction is expressed as ( $\hbar=1$ hereafter),

$$
\hat{H}=-\frac{1}{2 \mu_{R}} \frac{\partial^{2}}{\partial R^{2}}+\frac{\left(\hat{J}_{t o t}-\hat{J}\right)^{2}}{2 \mu_{R} R^{2}}+\hat{K}_{\mathrm{CH}_{3} \mathrm{Cl}}^{v i b}+\hat{K}_{\mathrm{CH}_{3} \mathrm{Cl}}^{r o t}+V(R, r, \rho, \gamma, \theta, \varphi),
$$

where $\mu_{R}$ is the reduced mass of the $\mathrm{F}^{-}+\mathrm{CH}_{3} \mathrm{Cl}$ system. $\hat{J}_{\text {tot }}$ is the total angular momentum operator of the system and $\hat{J}$ is the rotational angular momentum operator of $\mathrm{CH}_{3} \mathrm{Cl} . \hat{K}_{\mathrm{CH}_{3} \mathrm{Cl}}^{\mathrm{vb}}$ and $\hat{K}_{\mathrm{CH}_{3} \mathrm{Cl}}^{\text {rot }}$ are the vibrational and rotational kinetic energy operators of $\mathrm{CH}_{3} \mathrm{Cl}$, respectively. $V(R, r, \rho, \gamma, \theta, \varphi)$ is the potential energy. ${ }^{12}$

The vibrational and rotational kinetic energy operators of $\mathrm{CH}_{3} \mathrm{Cl}$ are expressed as, 


$$
\hat{K}_{\mathrm{CH}_{3} \mathrm{Cl}}^{v i b}=-\frac{1}{2 \mu_{r}} \frac{\partial^{2}}{\partial r^{2}}-\frac{1}{2 \mu_{x}}\left(\frac{\partial^{2}}{\partial q^{2}}+\frac{1}{q^{2}} \frac{\partial^{2}}{\partial \gamma^{2}}+\frac{1}{4 q^{2}}\right)
$$

where $\mu_{r}$ is the $\mathrm{CH}_{3}-\mathrm{Cl}$ reduced mass and $\mu_{x}=3 m_{H}$. The rotation of $\mathrm{CH}_{3} \mathrm{Cl}$ is describe by a symmetric-rotor Hamiltonian with $C_{3 \mathrm{v}}$ symmetric axis $\boldsymbol{S}$ as the z-axis

$$
\hat{K}_{\mathrm{CH}_{3} \mathrm{Cl}}^{\mathrm{rot}}=\frac{1}{2 I_{A}} \hat{J}^{2}+\left(\frac{1}{2 I_{C}}-\frac{1}{2 I_{A}}\right) \hat{J}_{z}^{2}
$$

where $\hat{J}_{z}$ is the $z$-component of $\hat{J}$. The moments of inertia $I_{A}$ and $I_{C}$ are defined as

$$
I_{A}=\frac{m_{C l}\left(m_{C}+3 m_{H}\right)}{m_{C l}+m_{C}+3 m_{H}} r^{2}+\frac{3 m_{C} m_{H}}{m_{C}+3 m_{H}} s^{2}+\frac{3}{2} m_{H} x^{2}
$$

and

$$
I_{C}=3 m_{H} x^{2}
$$

The time-dependent wave function is expanded in terms of the parity-adapted BF rotational basis functions $\Phi_{J k}^{J_{t o t} M K \varepsilon}$ as

$$
\Psi^{J_{\text {tot }} M \varepsilon}=\sum_{n_{R}, n_{r}, n_{q}, n_{\gamma}} \sum_{K J k} c_{n_{R} n_{r} n_{q} n_{\gamma} J k}^{J_{t o} M K \varepsilon}(t) G_{n_{R}}(R) F_{n_{r}}(r) Q_{n_{q}}(q) H_{n_{\gamma}}(\gamma) \Phi_{J k}^{J_{\text {tot }} M K \varepsilon}(\hat{R}, \hat{S}),
$$

and

$$
\Phi_{J k}^{J_{t o t} M K \varepsilon}=\sqrt{\frac{1}{2\left(1+\delta_{\bar{K} 0} \delta_{k 0}\right)}}\left[\bar{D}_{M \bar{K}}^{J_{t o t}} \bar{D}_{\bar{K} k}^{J}+\varepsilon(-1)^{J_{t o t}+k} \bar{D}_{M-\bar{K}}^{J_{t o t}} \bar{D}_{-\bar{K}-k}^{J}\right],
$$

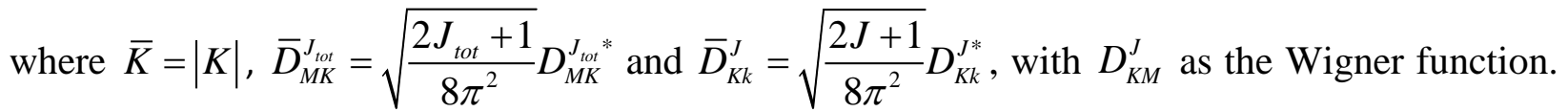
$C_{n_{R} n_{r} n_{q} n_{\gamma} J k}^{J_{t o} M K \varepsilon}(t)$ are time-dependent coefficients. $n_{R}, n_{r}, n_{q}$ and $n_{\gamma}$ are labels for the basis functions of $R, r, q$, and $\gamma$, respectively. $G_{n_{R}}(R)$ denote the sine basis functions, and the basis functions $F_{n_{r}}(r), Q_{n_{q}}(q)$, and $H_{n_{\gamma}}(\gamma)$ can be obtained by solving one-dimensional reference Hamiltonians. $J_{\text {tot }}$ is total angular momentum number of the system and $\varepsilon$ is space-inversion parity. $M$ and $K$ are the projections of $J_{\text {tot }}$ on the space fixed (SF) and the BF $z$-axis, respectively. 
$J$ is the angular momentum quantum number of $\mathrm{CH}_{3} \mathrm{Cl}$ and $k$ is its projection of $J$ on the $C_{3 \mathrm{v}}$ symmetry axis.

The initial wave packet is constructed as the direct product of a localized wave packet $G^{0}(R)$ and the eigenfunction of $\mathrm{CH}_{3} \mathrm{Cl}$ specified by $\left(n_{0}, J_{0}, k_{0}, K_{0}, p_{0}\right)$, which represent the initial vibrational quantum number, initial rotational quantum number of $\mathrm{CH}_{3} \mathrm{Cl}$, projection of $J_{0}$ on the $C_{3 \mathrm{v}}$ symmetry axis, projection of $J_{\text {tot }}$ on the $\mathrm{BF}$ z-axis, and parity of $\mathrm{CH}_{3} \mathrm{Cl}$, respectively. $G^{0}(R)$ is chosen to be a Gaussian function,

$$
G^{0}(R)=\left(\pi \delta^{2}\right)^{-1 / 4} \exp \left[-\frac{\left(R-R_{0}\right)^{2}}{2 \delta^{2}}\right] \exp \left(-i \sqrt{2 \mu_{R} E_{0}} R\right)
$$

where $R_{0}$ and $\delta$ are the center and width of the Gaussian function, and $E_{0}$ is the mean collision energy. The wave packet is propagated using the split operator method. ${ }^{13}$ The reaction probabilities for $J_{\text {tot }}>0$ partial waves are calculated with centrifugal sudden (CS) approximation. $^{14,} 15$ An absorbing potential, $\quad F_{a b s}=-i \alpha\left(\frac{x-x_{a}}{x_{\max }-x_{a}}\right)^{n}, \quad x_{a} \leq x \leq x_{\max } \quad$ is implemented at the edge of the grid to avoid artificial reflections. In this work, we are interested in the vibrational mode specificity and thus no rotationally excited $\mathrm{CH}_{3} \mathrm{Cl}$ is considered.

Numerical parameters of the quantum dynamical computations, which are carefully tested to give converged results, are given in Table S1. An $L$-shaped grid scheme is employed in numerical calculations. ${ }^{16}$ For the scattering coordinate $R, 1200$ sine discrete variable representation (DVR) ${ }^{17}$ basis/points are used in the whole range from 3.0 to $45.0 a_{0}$ and 200 sine DVR basis/points in the interaction region. For the dissociating $\mathrm{C}-\mathrm{Cl}$ bond of $r$, 50 potentialoptimized DVR (PODVR) ${ }^{17}$ basis/points are used in the interaction region and 7 PODVR basis/points in the asymptotic region. 6 and 20 PODVR basis/points are used along the scaledpolar coordinates $q$ and $\gamma$, respectively. The angular basis is given by $j_{\max }=120$ and $k_{\max }=6$. The center of the initial wave packet is located at $40.0 a_{0}$ and the propagation time is around 500 000 a.u., with a time step of 20 a.u. The flux dividing surface is located at $r^{F}=4.2 a_{0}$. 


\section{S-I B. Quasiclassical Trajectory Method}

QCT computations are performed for the $\mathrm{F}^{-}+\mathrm{CH}_{3} \mathrm{Cl}\left(v_{k}=0,1, J=0\right)$ reactions, where $k=1-6$, on the same PES $^{12}$ utilized in the QD computations. Standard normal-mode sampling ${ }^{18}$ is used to prepare the initial vibrational states. The mode-specific cross sections are obtained at several collision energies $\left(E_{\text {coll }}\right)$ by integration of the reaction probabilities over impact parameters based on roughly 200000 trajectories for each $E_{\text {coll }}$. Specific parameters of these computations are given in Table S2. At zero impact parameter, additional 50000 trajectories are computed in order to get improved statistics for QD vs. QCT comparison at $J_{\text {tot }}=0$.

Direct and indirect trajectories are separated based on the integration time. Direct trajectories finish fast within a narrow time interval; however, this time range depends on the relative velocity (collision energy) and initial distance of the reactants. Therefore, we have examined the integration time distribution at each collision energy, and found that 1.74, 1.23, $0.87,0.73$, and $0.65 \mathrm{ps}$ time limits separate the direct and indirect trajectories at collision energies of 1, 2, 4, 7, and $10 \mathrm{kcal} / \mathrm{mol}$, respectively. Below these limits the direct trajectories feature a sharp peak in the integration time distributions, whereas the indirect trajectories correspond to broad distributions ranging from the above time limits to hundreds of picoseconds.

\section{S-I C. Sudden Vector Projection Model}

The SVP model ${ }^{19}$ is based on the assumption that the intramolecular vibrational energy redistribution (IVR) of reactants is much slower than the collision time. This assumption is only fulfilled in the direct channel of $S_{N} 2$ reactions. In the SVP model, the efficacy of a reactant mode in promoting the reaction is attributed to its coupling with the reaction coordinate at the transition state. In this reaction, the transition state is defined by the submerged central saddle point on the PES. The coupling is approximated by the projection of the reactant normal mode vector $\left(\vec{Q}_{i}\right)$ onto the reaction coordinate vector $\left(\vec{Q}_{R C}\right): \eta_{i}=\vec{Q}_{i} \cdot \vec{Q}_{R C} \in[0,1]$. The SVP model has been successfully applied to predict the mode specificity and bond selectivity in many polyatomic reactions. ${ }^{20}$ In Table S3, the SVP values are listed for the translational and all vibrational modes of $\mathrm{CH}_{3} \mathrm{Cl}$ with regard to the Walden-inversion saddle point. 


\section{S-II. Additional Results}

In Figure S2, the $\left(J_{t o t}=0\right)$ reaction probabilities for eight low-lying vibrational states of the $\mathrm{CH}_{3} \mathrm{Cl}$ reactant are shown as a function of collision energy. They all behave roughly the same, underscoring the common reaction mechanisms as discussed in the main text. In the same figure, the ratios of the reaction probabilities of the vibrationally excited reactants to that of the ground state are also presented. Apart from the mode specificity of fundamentally excited reactant, it is also interesting to investigate the reactivity for combination excitations. The enhancement of reactivity of a combined excitation appears to be roughly the sum of the individual excitations, with little synergistic effect. The results are different from those obtained by Hennig and Schmatz on $\mathrm{Cl}^{-}+\mathrm{CH}_{3} \mathrm{Cl}^{\prime}$ and $\mathrm{Cl}^{-}+\mathrm{CH}_{3} \mathrm{Br}$ reactions, in which 4D QD calculations found significant synergistic effects. ${ }^{21,22}$ In addition, the excitation in the first overtone of the $\mathrm{CH}_{3}$ umbrella mode $\left(2 v_{2}\right)$ has a similar effect to the fundamental excitation $\left(v_{2}\right)$ over the entire energy range except at very low and very high collision energies.

While the QD calculations in the main text yielded the integral cross section (ICS) for the title reaction with the $\mathrm{CH}_{3} \mathrm{Cl}$ in its ground vibrational state, the computational costs are too great to perform similar calculations for the vibrational excited states of the $\mathrm{CH}_{3} \mathrm{Cl}$ reactant. Instead, these ICSs are computed using the full-dimensional QCT computations. In Figure S3 we present the mode-specific ICS ratios as a function of collision energy for all the vibrational modes of the reactant. Vibrational enhancement factors usually increase with $E_{\text {coll }}$ and larger effects are seen than in the zero impact parameter case. The $\mathrm{C}-\mathrm{Cl}$ stretching mode has the largest enhancement effect for the entire collision energy range investigated in this work, which is in excellent agreement with the SVP prediction. On one hand, umbrella mode excitation usually promotes the reaction more efficiently than the other two bending modes, which have higher frequencies but weaker couplings to the reaction coordinate (Table S3). On the other hand, umbrella excitation has a smaller promotion effect than $\mathrm{C}-\mathrm{Cl}$ stretching excitation, in good agreement with the QD findings.

It should be bear in mind that the ratios in Figure S3 include contributions from both direct and indirect channels. The direct channel is dominated by relatively fast dynamics. However, trajectories can be trapped in the hydrogen-bonded and/or ion-dipole wells before and 
after the central barrier in the indirect mechanism. If the lifetime of a trapped trajectory is sufficiently long, energy randomization might occur due to IVR. The IVR in classical dynamics is often faster than that in a quantum system, and this "unphysical" energy randomization might help the $\mathrm{CH}$ stretching excited reactant to channel its vibrational energy $\left(\sim 3000 \mathrm{~cm}^{-1}\right)$ into the reaction coordinate, thus enhancing reactivity fictitiously. These are of course speculations that require verification by quantum mechanical studies in the future.

To gain further insight into the dependence of mode specificity with the reaction mechanism, the fraction of the direct trajectories is plotted in Figure S4 for these reactant states. It can be seen that while the fraction of direct trajectories generally increases with energy, it is still relatively small. In the same figure, the QCT ratios are plotted for the direct trajectories. It is clear that in the direct channel the $\mathrm{C}$ - $\mathrm{Cl}$ vibrational excitation enhances the reaction most significantly. The $\mathrm{CH}$ stretching excitation still maintains some enhancement, but with lesser extent than in the case of the indirect trajectories. In the case of the direct trajectories the umbrella excitation effect supercedes the $\mathrm{CH}$ stretching effect. These findings are in general consistent with QD results and the SVP predictions.

\section{References:}

(1) Palma, J.; Clary, D. C. A Quantum Model Hamiltonian to Treat Reactions of the Type $\mathrm{X}+\mathrm{YCZ} \mathrm{Z}_{3} \rightarrow$ $\mathrm{XY}+\mathrm{CZ}_{3}$ : Application to $\mathrm{O}\left({ }^{3} \mathrm{P}\right)+\mathrm{CH}_{4} \rightarrow \mathrm{OH}+\mathrm{CH}_{3}$, J. Chem. Phys. 2000, 112, 1859-1867.

(2) Yang, M.; Zhang, D. H.; Lee, S.-Y. A Seven-Dimensional Quantum Study of the $\mathrm{H}+\mathrm{CH}_{4}$ Reaction, J. Chem. Phys. 2002, 117, 9539.

(3) Yang, M.; Lee, S.-Y.; Zhang, D. H. Seven-Dimensional Quantum Dynamics Study of the O( $\left.{ }^{3} \mathrm{P}\right)+\mathrm{CH}_{4}$ Reaction, J. Chem. Phys. 2007, 126, 064303.

(4) Zhou, Y.; Wang, C.; Zhang, D. H. Effects of Reagent Vibrational Excitation on the Dynamics of the $\mathrm{H}+\mathrm{CHD}_{3} \rightarrow \mathrm{H}_{2}+\mathrm{CD}_{3}$ Reaction: A Seven-Dimensional Time-Dependent Wave Packet Study, J. Chem. Phys. 2011, 135, 024313.

(5) Zhang, Z.; Zhou, Y.; Zhang, D. H.; Czakó, G.; Bowman, J. M. Theoretical Study of the Validity of the Polanyi Rules for the Late-Barrier $\mathrm{Cl}+\mathrm{CHD}_{3}$ Reaction, J. Phys. Chem. Lett. 2012, 3, 34163419.

(6) Liu, R.; Xiong, H.; Yang, M. An Eight-Dimensional Quantum Mechanical Hamiltonian for $\mathrm{X}+\mathrm{YCZ} \mathrm{CZ}_{3}$ System and Its Applications to $\mathrm{H}+\mathrm{CH}_{4}$ Reaction, J. Chem. Phys. 2012, 137, 174113.

(7) Liu, R.; Yang, M.; Czakó, G.; Bowman, J. M.; Li, J.; Guo, H. Mode Selectivity for a "Central" Barrier Reaction: Eight-Dimensional Quantum Studies of the $\mathrm{O}\left({ }^{3} \mathrm{P}\right)+\mathrm{CH}_{4} \rightarrow \mathrm{OH}+\mathrm{CH}_{3}$ Reaction on an $\mathrm{Ab}$ Initio Potential Energy Surface, J. Phys. Chem. Lett. 2012, 3, 3776-3780.

(8) Liu, R.; Wang, F.; Jiang, B.; Czakó, G.; Yang, M.; Liu, K.; Guo, H. Rotational Mode Specificity in the $\mathrm{Cl}+\mathrm{CHD}_{3} \rightarrow \mathrm{HCl}+\mathrm{CD}_{3}$ Reaction, J. Chem. Phys. 2014, 141, 074310. 
(9) Zhang, Z.; Zhang, D. H. Effects of Reagent Rotational Excitation on the $\mathrm{H}+\mathrm{CHD}_{3} \rightarrow \mathrm{H}_{2}+\mathrm{CD}_{3}$ Reaction: A Seven Dimensional Time-Dependent Wave Packet Study, J. Chem. Phys. 2014, 141, 144309

(10) Su, T.; Wang, H.; Hase, W. L. Trajectory Studies of $\mathrm{S}_{\mathrm{N}} 2$ Nucleophilic Substitution. 7. $\mathrm{F}^{-}+\mathrm{CH}_{3} \mathrm{Cl} \rightarrow$ $\mathrm{FCH}_{3}+\mathrm{Cl}^{-}$, J. Phys. Chem. A 1998, 102, 9819-9828.

(11) Szabó, I.; Császár, A. G.; Czakó, G. Dynamics of the $\mathrm{F}^{-}+\mathrm{CH}_{3} \mathrm{Cl} \rightarrow \mathrm{Cl}^{-}+\mathrm{CH}_{3} \mathrm{~F} \mathrm{~S}_{\mathrm{N}} 2$ Reaction on a Chemically Accurate Potential Energy Surface, Chem. Sci. 2013, 4, 4362-4370.

(12) Szabó, I.; Czakó, G. Revealing a Double-Inversion Mechanism for the $\mathrm{F}^{-}+\mathrm{CH}_{3} \mathrm{Cl} \mathrm{S}_{\mathrm{N}} 2$ Reaction, Nat. Commun. 2015, 6, 5972.

(13) Feit, M. D.; Fleck, J. A. Solution of the Schrodinger Equation by a Spectral Method II: Vibrational Energy Levels of Triatomic Molecules, J. Chem. Phys. 1983, 78, 301.

(14) McGuire, P.; Kouri, D. J. Quantum Mechanical Close Coupling Approach to Molecular Collisions. $\mathrm{J}_{\mathrm{z}}$-Conserving Coupled States Approximation, J. Chem. Phys. 1974, 60, 2488-2499.

(15) Pack, R. T. Space-Fixed Vs Body-Fixed Axes in Atom-Diatomic Molecule Scattering. Sudden Approximations, J. Chem. Phys. 1974, 60, 633-639.

(16) Zhang, D. H.; Zhang, J. Z. H. Accurate Quantum Calculations for the Benchmark Reaction $\mathrm{H}_{2}+\mathrm{OH}$ $\rightarrow \mathrm{H}_{2} \mathrm{O}+\mathrm{H}$ in Five-Dimensional Space: Reaction Probability for J=0, J. Chem. Phys. 1993, 99, 5615-5618.

(17) Light, J. C.; Carrington Jr., T. Discrete-Variable Representations and Their Utilization, Adv. Chem. Phys. 2000, 114, 263-310.

(18) Hase, W. L. (1998) Classical Trajectory Simulations: Initial Conditions, In Encyclopedia of Computational Chemistry (Alinger, N. L., Ed.), pp 399-402, Wiley, New York.

(19) Jiang, B.; Guo, H. Relative Efficacy of Vibrational Vs. Translational Excitation in Promoting AtomDiatom Reactivity: Rigorous Examination of Polanyi's Rules and Proposition of Sudden Vector Projection (SVP) Model, J. Chem. Phys. 2013, 138, 234104.

(20) Guo, H.; Jiang, B. The Sudden Vector Projection Model for Reactivity: Mode Specificity and Bond Selectivity Made Simple, Acc. Chem. Res. 2014, 47, 3679-3685.

(21) Hennig, C.; Schmatz, S. State-Selected Dynamics of the Complex-Forming Bimolecular Reaction $\mathrm{Cl}^{-}+\mathrm{CH}_{3} \mathrm{Cl}^{\prime} \rightarrow \mathrm{ClCH}_{3}+\mathrm{Cl}^{-}$: A Four-Dimensional Quantum Scattering Study, J. Chem. Phys. 2004, 121, 220-236.

(22) Hennig, C.; Schmatz, S. Four-Dimensional Quantum Study on Exothermic Complex-Forming Reactions: $\mathrm{Cl}^{-}+\mathrm{CH}_{3} \mathrm{Br} \rightarrow \mathrm{ClCH}_{3}+\mathrm{Br}^{-}$, J. Chem. Phys. 2005, 122, 234307. 
Table S1. Numerical parameters used in the wave packet calculations. (Atomic units are used unless stated otherwise.)

\begin{tabular}{cc}
\hline \hline & $\mathrm{F}^{-}+\mathrm{CH}_{3} \mathrm{Cl} \rightarrow \mathrm{CH}_{3} \mathrm{~F}+\mathrm{Cl}^{-}$ \\
& $R \in[3.0,45.0]$ \\
& $r \in[1.0,6.0]$ \\
Grid/basis & $N_{R}{ }^{t o t}=1200, N_{R}{ }^{i n t}=200$ \\
range and size: & $N_{r}{ }^{i n t}=50, N_{r}{ }^{a s y}=7$ \\
& $N_{q}=6, N_{\gamma}=20$ \\
& $J_{\max }=120, k_{\text {max }}=6$ \\
packet: & $R_{0}=40.0, \delta=0.09, E_{0}=0.26 \mathrm{eV}$ \\
\hline Absorbing potential: & $R_{a}=42.2, \alpha_{R}=0.025, n_{R}=1.5$ \\
& $r_{a}=4.5, \alpha_{r}=0.020, n_{r}=1.5$ \\
\hline Flux position: & 500,000 \\
\hline Propagation time & $r^{F}=4.2$ \\
\hline \hline
\end{tabular}


Table S2. Settings for the initial distance of the reactants and impact parameter scans (in bohr) and the total number of trajectories at each collision energy (in $\mathrm{kcal} \mathrm{mol}^{-1}$ ). ${ }^{a}$

\begin{tabular}{ccccc}
\hline \hline$E_{\text {coll }}$ & $x$ & $\Delta b$ & $b_{\max }{ }^{b}$ & $N_{\text {traj }}^{b}$ \\
\hline 0.5 & 35 & 1.0 & 32 & 165000 \\
1.0 & 30 & 1.0 & 26 & 135000 \\
2.0 & 25 & 0.5 & 21 & 215000 \\
4.0 & 20 & 0.5 & 18 & 185000 \\
7.0 & 20 & 0.5 & 16 & 165000 \\
10.0 & 20 & 0.5 & 14 & 145000 \\
\hline \hline
\end{tabular}

${ }^{a}$ The initial distance of the reactants is $\sqrt{x^{2}+b^{2}}$, where $b=0, \Delta b, 2 \Delta b, \ldots, b_{\max }$ and $N_{\text {traj }}=$ $\left(b_{\max } / \Delta b+1\right) \times 5000$.

${ }^{b} b_{\max }$ and $N_{\text {traj }}$ slightly vary for the different initial vibrational states at a given $E_{\text {coll }}$, here the largest values are given for each $E_{\text {coll }}$. 
Table S3. SVP values of the translational and vibrational modes of $\mathrm{CH}_{3} \mathrm{Cl}$ for the direct $\mathrm{F}^{-}+$ $\mathrm{CH}_{3} \mathrm{Cl} \rightarrow \mathrm{CH}_{3} \mathrm{~F}+\mathrm{Cl}^{-}$reaction.

\begin{tabular}{c|c}
\hline \hline Normal Mode & SVP \\
\hline $\mathrm{CH}_{3}$ s-str, $\left(v_{1}\right)$ & 0.10 \\
$\mathrm{CH}_{3}$ s-deform, $\left(v_{2}\right)$ & 0.33 \\
$\mathrm{CCl}$ str, $\left(v_{3}\right)$ & 0.84 \\
$\mathrm{CH}_{3}$ d-str $\left(v_{4}\right)$ & 0.00 \\
$\mathrm{CH}_{3}$ d-deform $\left(v_{5}\right)$ & 0.01 \\
$\mathrm{CH}_{3}$ rock $\left(v_{6}\right)$ & 0.01 \\
$\operatorname{trans}$ & 0.42 \\
\hline \hline
\end{tabular}




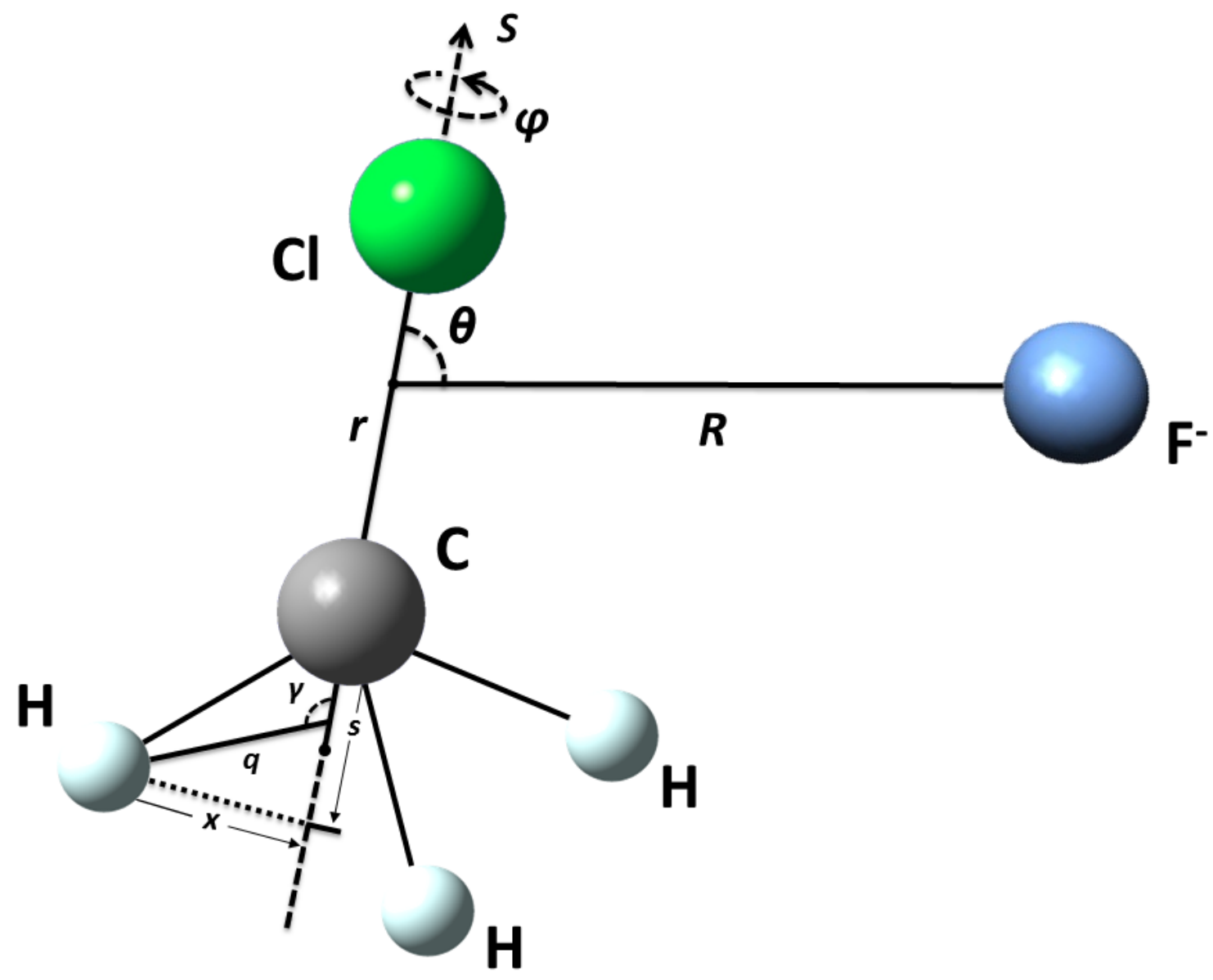

Figure S1. The six-dimensional reactant Jacobi coordinates for the $\mathrm{X}^{-}+\mathrm{CH}_{3} \mathrm{Y}$ system used in the QD calculations. 

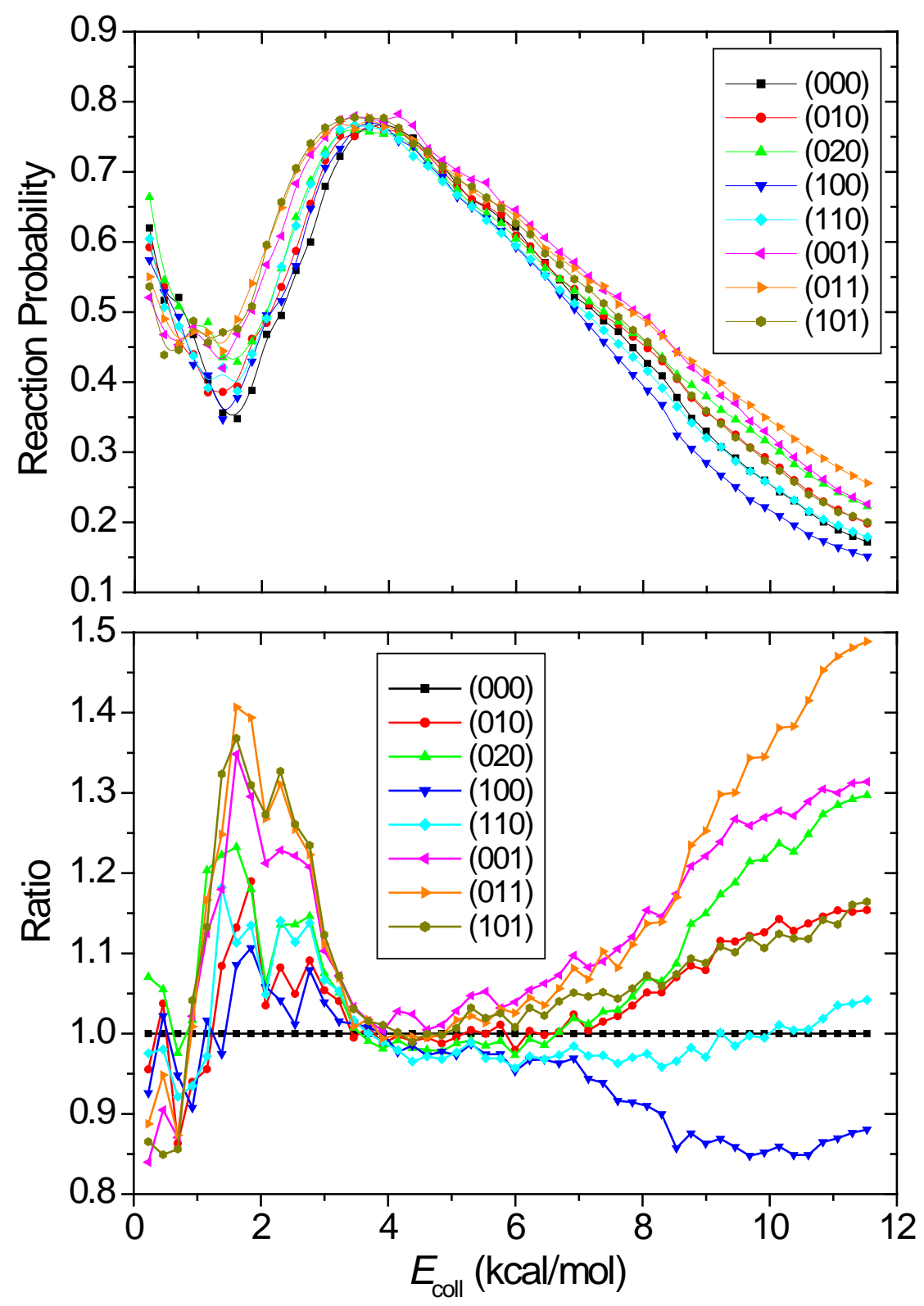

Figure S2. QD reaction probabilities and their ratios from several selected initial states, labeled by $\left(v_{1}, v_{2}, v_{3}\right)$, for the $\mathrm{F}^{-}+\mathrm{CH}_{3} \mathrm{Cl} \rightarrow \mathrm{CH}_{3} \mathrm{~F}+\mathrm{Cl}^{-}$reaction with the total angular momentum $J_{\text {tot }}$ $=0$ as a function of collision energy. 


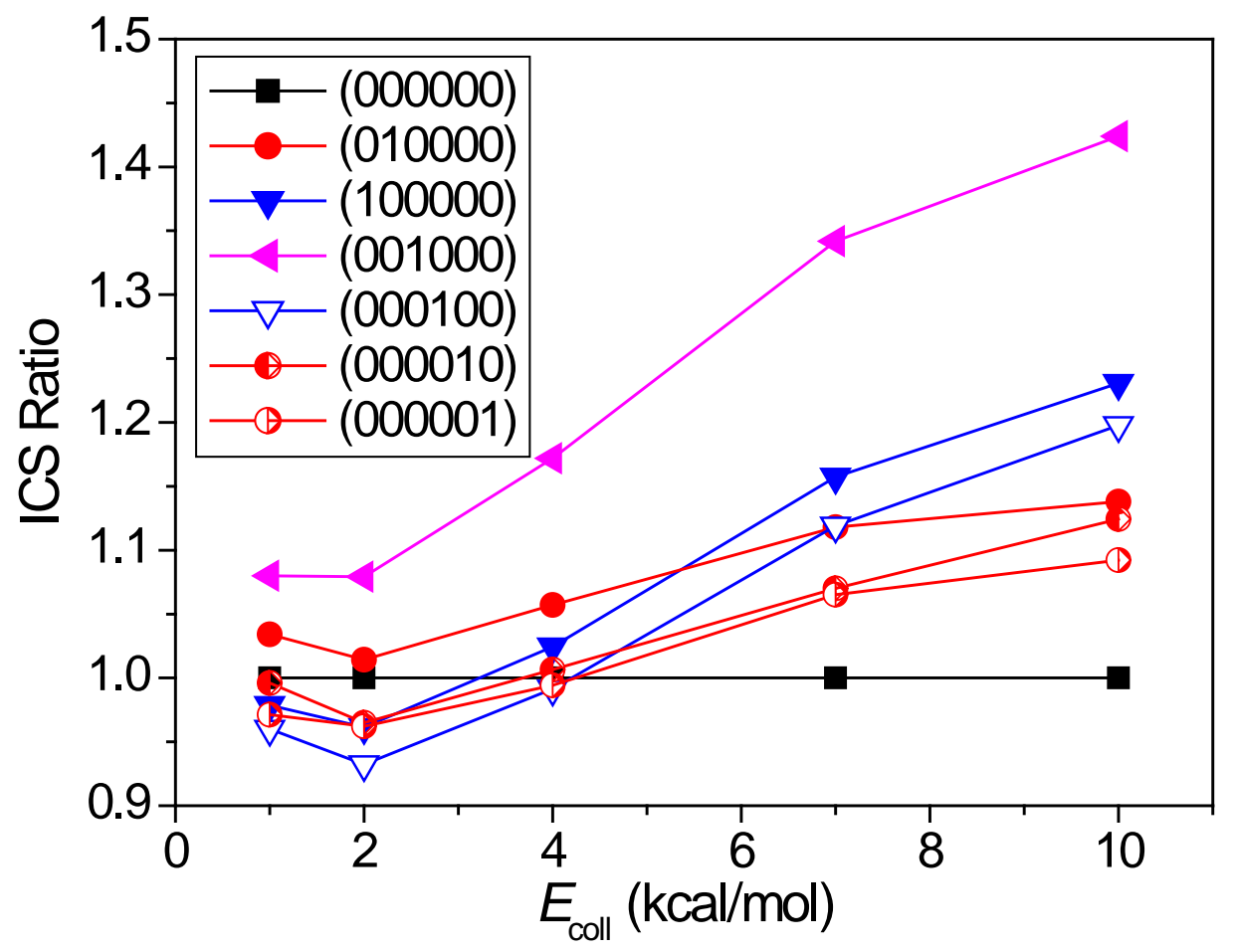

Figure S3. QCT cross section ratios for all the fundamental initial vibrational states, labeled by $\left(v_{1}, v_{2}, v_{3}, v_{4}, v_{5}, v_{6}\right)$, of the $\mathrm{F}^{-}+\mathrm{CH}_{3} \mathrm{Cl} \mathrm{S} \mathrm{N}_{2}$ reaction as a function of collision energy. 

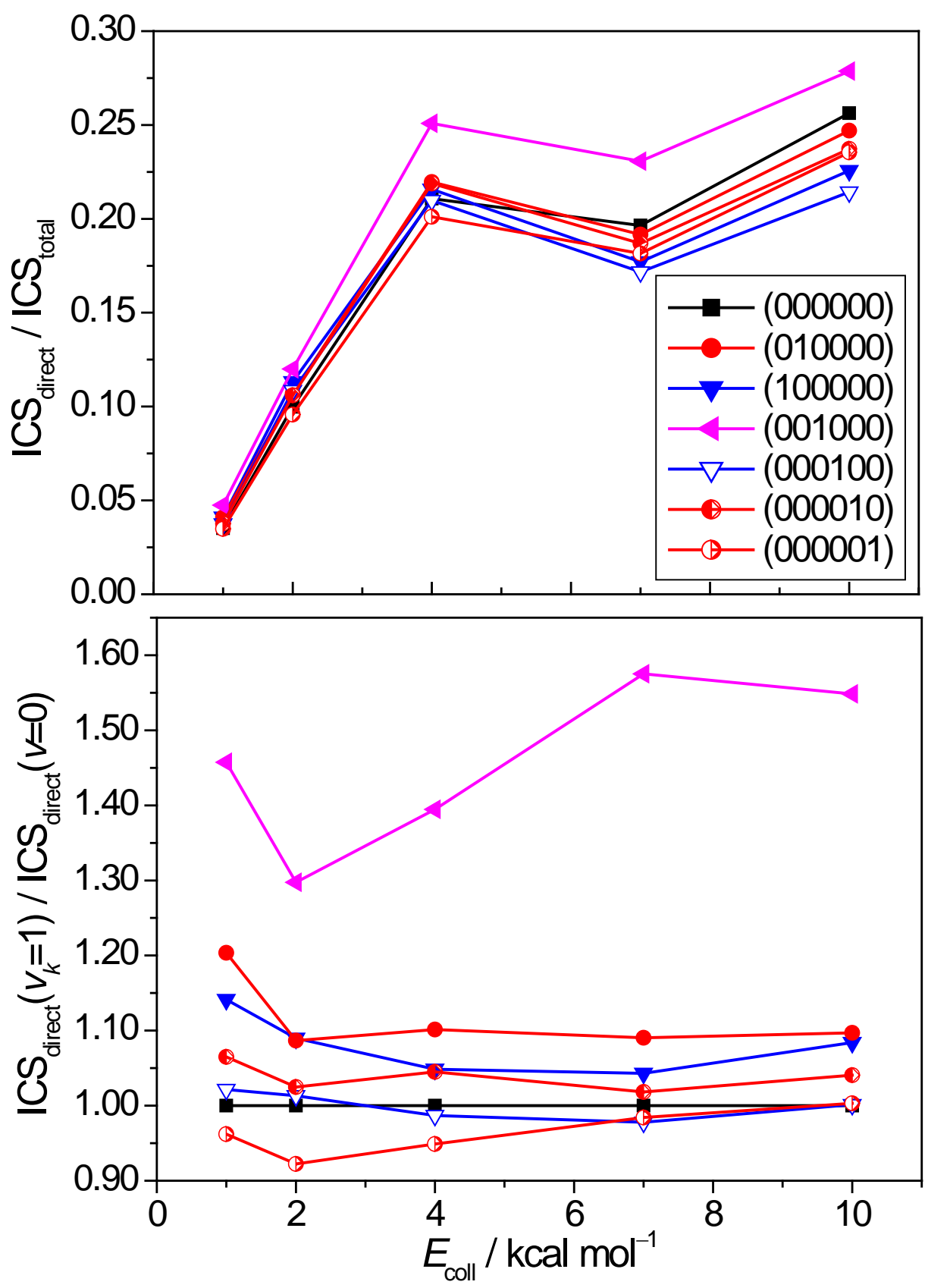

Figure S4. The component of direct QCT cross section in the total cross section and cross section ratios for the direct reaction from all the fundamental initial vibrational states, labeled by $\left(v_{1}, v_{2}, v_{3}, v_{4}, v_{5}, v_{6}\right)$, of the $\mathrm{F}^{-}+\mathrm{CH}_{3} \mathrm{Cl} \mathrm{S}_{\mathrm{N}} 2$ reaction as a function of collision energy. 